\title{
12 What can INFER do?
}

INFER carries out significance tests and calculates confidence limits for univariate data. Data may be entered at the keyboard or retrieved from a data file created by FILER (except for analysis of variance where only keyboard data entry is available).

INFER can be used to:

(1) Test a single sample $-Z$ test and t test.

(2) Test a paired sample - Paired sample t test and Wilcoxon signed-rank test.

(3) Test two independent samples - F test, Unpaired (standard) $t$ test, Unpaired (modified) $t$ test and MannWhitney test.

(4) Carry out One-factor analysis of variance, Two-factor analysis of variance, the Kruskal-Wallis test and the Friedman test. Missing values are not allowed in Twofactor analysis of variance.

For all the above tests, the data and results can be sent to a printer. The data can also be edited.

\section{What can BIVAR do?}

BIVAR performs correlation and regression analysis for bivariate data. Data may be entered at the keyboard or retrieved from a data file created by FILER. It is also possible to select any two variables from a multivariate file created by FILER.

BIVAR can be used to:

(1) List the $X$ and $Y$ values. These will always be your latest 